\title{
A 16-week randomized controlled trial evaluating the physical activity guidelines for adults with spinal cord injury
}

\author{
CA Pelletier, JO Totosy de Zepetnek, MJ MacDonald and AL Hicks
}

Study Design: Randomized controlled trial.

Objectives: To evaluate the effectiveness of the physical activity guidelines (PAG) for adults with spinal cord injury (SCI) to improve aspects of physical fitness.

Setting: Community exercise facility.

Methods: Twenty-three participants (age: $40.4 \pm 11.6$ years, C1-T11, $12.0 \pm 10.0$ years post injury) were randomized into PAG training $(n=12)$ or active control (CON, $n=11)$ groups. PAG training was $2 x$ per week for 16 weeks and involved 20 min of aerobic exercise at a moderate to vigorous intensity and three sets of 10 repetitions (at 50-70\% 1 repetition maximum; 1RM). Pre- and post-testing included peak exercise and aerobic endurance tests on an arm ergometer and 1RM testing.

Results: Nineteen participants (PAG, $n=11 ; \mathrm{CON}, n=8$ ) completed the 16 -week training program and post-testing. There was a significant $(P<0.05)$ increase in peak aerobic capacity (relative $\mathrm{VO}_{2 \text { peak }}: 17.2 \%$, absolute $\mathrm{VO}_{2 \text { peak: }} 9.9 \%$ ) and submaximal power output $(26.3 \%)$ in the PAG group only. Increases in strength ranged from $11.5-38.9 \%$ and were significantly $(P<0.05)$ different from CON for vertical bench press, seated row, and rickshaw press. Adherence to the exercise program was $85.2 \pm 8.3 \%$ for PAG, $44.4 \pm 34.3 \%$ for $\operatorname{CON}(P<0.01)$.

Conclusion: The PAG for adults with $\mathrm{SCl}$ are sufficient to improve aspects of aerobic and muscular fitness and should be promoted as a means to improve physical capacity.

Sponsorship: Ontario Neurotrauma Foundation (ONF), Natural Sciences and Engineering Research Council (NSERC) of Canada. Spinal Cord (2015) 53, 363-367; doi:10.1038/sc.2014.167; published online 30 September 2014

\section{INTRODUCTION}

Cardiovascular disease is the leading cause of mortality among persons with spinal cord injury (SCI). ${ }^{1}$ In the able-bodied population, physical inactivity is an independent risk factor for cardiovascular disease and has indirect effects through its impact on body mass index, glucose homeostasis and blood lipids. Physical fitness is directly related to physical activity, and is also linked to many aspects of quality of life in the SCI population. ${ }^{2}$ Thus, there is an urgent need for health promotion strategies to encourage the adoption of regular physical activity.

The Canadian physical activity guidelines (PAG) for adults with SCI were released in 2011 in order to provide clinicians and exercise professionals information from which to form an exercise prescription specific to this population. ${ }^{3}$ The evidence-based guidelines, based on a systematic review and expert panel consensus meeting, ${ }^{3,4}$ recommend exercise twice weekly involving aerobic activity at a moderate to vigorous intensity for at least $20 \mathrm{~min}$, and resistance activity of three sets of 10 repetitions using each major muscle group.

There is strong evidence to support the value of exercise to improve aspects of physical fitness in the SCI population. Improvements in cardiovascular fitness have been demonstrated following aerobic exercise training using arm cycle ${ }^{5}$ or wheelchair ergometry, ${ }^{6}$ and improvements in muscle strength have been demonstrated with both neuromuscular electrical stimulation ${ }^{7}$ and voluntary muscle contraction training protocols. ${ }^{8}$ Few studies have demonstrated the efficacy of combined aerobic and resistance exercise training programs in the SCI population. A 9-month twice weekly training protocol of combined resistance (2-3 sets of $50-80 \% 1$ repetition maximum (1RM)) and aerobic exercise (70\% maximum heart rate or 3-4 on 10-point rating of perceived exertion (RPE) scale) resulted in an increase of $81 \%$ in submaximal power output, and a $19-34 \%$ increase in muscle strength. ${ }^{9}$ Circuit training (arm cycle ergometry and resistance training done in sequence) has also produced improvements of $10.4 \%$ in peak aerobic capacity and increases in muscle strength of 38.6-59.7\% after 4 months of training three times per week. ${ }^{10}$

Now that evidence-based PAG have been developed, an important next step is to implement the guidelines in a practical community setting to establish their validity in improving physical fitness in adults with chronic SCI. Thus, the purpose of this study was to evaluate the effectiveness of the PAG for adults with SCI for improving aspects of fitness in a community-based sample. It was predicted that 16 weeks of training following the recommendations of the PAG would result in significant increases in aerobic fitness and muscle strength when compared with an active control group. 


\section{METHODS}

\section{Participants}

Participants with chronic SCI ( $>1$ year post injury), who were 18-65 years old, relied on a wheelchair for mobility, and were able to understand English were recruited to participate from April 2012 to August 2013 through advertisements at local community organizations. Exclusion criteria included any progressive loss of neurologic function within the previous 6 months. The Hamilton Health Sciences Research Ethics board approved the study protocol, and informed written consent was obtained from each participant. After baseline testing was completed, participants were randomized into a PAG training or active control (CON) group using computer software (GraphPad Software, La Jolla, CA, USA).

\section{Peak aerobic capacity}

To measure peak oxygen consumption $\left(\mathrm{VO}_{2 \text { peak }}\right)$, participants performed a symptom-limited, graded exercise test on an arm cycle ergometer (Lode B.V., Groningen, the Netherlands) at baseline (pre training) and 16 weeks (post training). Resistance was increased by $5 \mathrm{~W} \mathrm{~min}^{-1}$ for participants with tetraplegia and $10 \mathrm{~W} \mathrm{~min}{ }^{-1}$ for participants with paraplegia. ${ }^{11}$ In some cases, this protocol was modified to ensure the test was $8-12 \mathrm{~min}$ in duration. Participants were asked to continue until volitional fatigue or they were unable to maintain a cadence of 40 r.p.m. Expired gas and ventilatory parameters were acquired allowing for the determination of $\mathrm{VO}_{2 \text { peak }}$ (Moxus Metabolic System, AEI Technologies, Pittsburgh, PA, USA). Central and peripheral RPE (Borg 10point scale $)^{12}$ were assessed every minute. Heart rate (HR) was recorded throughout the protocol (Polar Electro, Lachine, QC, Canada). VO ${ }_{2 \text { peak }}, \mathrm{HR}_{\text {peak }}$ and peak minute ventilation were defined as the greatest value achieved in a 30$\mathrm{s}$ interval. Peak power output $\left(\mathrm{PO}_{\text {peak }}\right)$ was defined as the greatest resistance achieved and maintained for at least $15 \mathrm{~s}$.

\section{Aerobic endurance}

On a separate day, pre and post training, participants were asked to complete an aerobic endurance test on an arm cycle ergometer (Lode B.V.). After a 1-min warm-up, resistance (W) was set to the corresponding value achieved at $70 \%$ of $\mathrm{VO}_{2 \text { peak }}$. $\mathrm{HR}$ and $\mathrm{VO}_{2}$ were continuously monitored (Polar Electro; Moxus Metabolic System, AEI Technologies). Participants were instructed to continue until volitional fatigue (unable to maintain 40 r.p.m.) and the test was terminated at $20 \mathrm{~min}$.

\section{Muscle strength \\ Muscle strength testing was completed on a wheelchair accessible weight training machine (Equalizer Multi-Station, Equalizer Exercise Machines, Red Deer, AB, Canada) or unilateral wall pulleys (Endorphin Pulleys, Patterson Medical Supply, Mississauga, ON, Canada) pre and post training. 1RM was assessed in a variety of upper body exercises: latissimus pull-down, vertical bench press, seated row, bicep curl (right and left) and rickshaw press (elbow extension). The order of exercises was standardized, and there was sufficient rest between exercises to minimize the potential influence of muscle fatigue.}

\section{Training intervention}

Participants in the PAG group completed a 16-week supervised, progressive exercise program that followed the PAG for adults with SCI. The twice weekly program consisted of $20 \mathrm{~min}$ of aerobic exercise at a moderate to vigorous intensity (RPE 3-6 on 10-point scale) and three sets of 10 repetitions (50-70\% 1RM) of resistance exercise using each major muscle group of the upper body. The aerobic exercise equipment used was an arm cycle ergometer (Monark Rehab Trainer, Patterson Medical Supply) or hybrid recumbent stepper (NuStep T5XR Recumbent Cross Trainer, NuStep, Inc., Ann Arbor, MI, USA). Resistance exercise was completed using a combination of multi-station accessible weight stack equipment (Equalizer Multi-Station, Equalizer Exercise Machines), wall pulleys (Endorphin Pulleys, Patterson Medical Supply) and free weights.

Participants in the CON group were members in a twice weekly community exercise program geared for adults with SCI. They were given no specific guidance or encouragement with respect to attaining the minimum exercise intensity associated with the PAG.

\section{Adherence}

Adherence was calculated based on the percentage of a maximum of 32 sessions (2x per week for 16 weeks). If participants in the PAG group missed any sessions during the training period they were given 4 weeks to complete make-up sessions. Adherence rates are presented as (i) during the 16-week period (for comparison with $\mathrm{CON}$ ) and (ii) total number of sessions completed (including make-up sessions).

\section{Satisfaction with the guidelines}

A questionnaire to assess consumer satisfaction with the PAG was administered to participants in the PAG group post training. All questions were rated from $1=$ strongly disagree to $7=$ strongly agree, and mean composite scores were calculated for each set of items. Satisfaction with aerobic and resistance exercise was evaluated with five scales each, asking whether the participants felt the training program was appropriate in terms of time, intensity, ability, if it was enjoyable and if it improved their physical fitness. Perception of pain was measured with one item: 'I was able to complete the exercises without any additional pain or discomfort.'

\section{Data analysis}

All data are presented as mean \pm s.d. and were analyzed using IBM SPSS software (Version 20.0, IBM Corporation, Armonk, NY, USA). Participant demographic information, adherence rates and pre-training data were compared between the groups using a Student's $t$-test or the Welch F-test where the assumption of equal variances was violated. Pre- and post-training values were compared between the groups using a two-way (group $\mathrm{x}$ time) repeated measures analysis of variance and significance was accepted at $P<0.05$.

\section{RESULTS}

\section{Participants}

Figure 1 depicts the flow of participants. Twenty-three participants (age $40.4 \pm 11.6$ years, C1-T11, $12.0 \pm 10.0$ years post injury) were recruited to participate; 12 were randomized into the PAG group and 11 into the CON group. Four participants did not complete all aspects of the study: one dropped out immediately following randomization due to dissatisfaction with group allocation, two participants did not complete post-testing due to the re-occurrence of chronic injuries (not study related), and one participant dropped out due to an unrelated incident. There were no differences in demographic information between the groups (Table 1).

\section{Peak aerobic capacity}

Results of the peak exercise tests are presented in Table 2. The groups were similar at baseline $(P>0.05)$. There was a significant group by time interaction in relative $(P=0.01)$ and absolute $P=0.004 \mathrm{VO}_{2 \text { peak }}$, indicating increased aerobic capacity in the PAG group following training. While there was a $13.4 \%$ increase in $\mathrm{PO}_{\text {peak }}$ in the PAG group following training, it did not reach statistical significance $(P=0.059)$.

\section{Aerobic endurance}

There were no differences between groups at baseline for any of the variables $(P>0.05$, Table 3$)$. Post training, the PAG group completed the submaximal test at a higher power output than the CON group $(P=0.01)$.

\section{Muscle strength}

Changes in muscle strength are presented in Table 4. PAG and CON were well matched at baseline $(P>0.05)$. There was a significant group $\mathrm{x}$ time interaction for vertical bench press $(P=0.02)$, seated row $(P=0.03)$ and elbow extension $(P<0.01)$, reflective of mean strength 


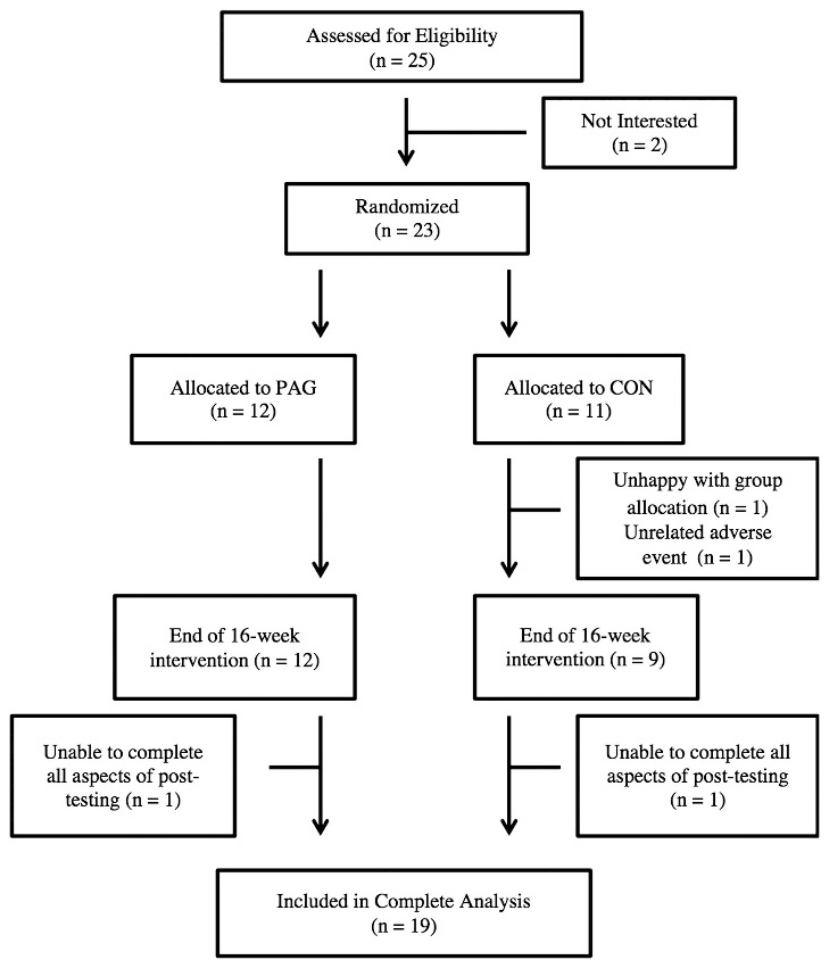

Figure 1 Flow chart of participants from recruitment through the end of the 16-week intervention.

increases in the PAG group of $7.3 \pm 6.4 \mathrm{~kg}, 8.3 \pm 6.1 \mathrm{~kg}$ and $22.5 \pm 23.1 \mathrm{~kg}$, respectively.

\section{Adherence}

Mean adherence rates were $85.2 \pm 8.3 \%$ (range $72-100 \%$ ) among participants in PAG and $44.4 \pm 34.3 \%$ (range $0-93.8 \%$ ) in CON $(P=0.01)$. When the make-up sessions were included, mean adherence was $98.4 \pm 3.3 \%$ in the PAG group. The most prevalent barriers in the PAG group were personal health issues $(80 \%)$, other commitments (18.8\%), transportation (12.5\%), medical appointments $(12.5 \%)$ and vacation $(6.3 \%)$.

\section{Satisfaction with the Guidelines}

Satisfaction with both the aerobic $(6.3 \pm 0.64)$ and resistance $(6.7 \pm 0.5)$ aspects of the PAG training protocol were high (maximum score of 7). Enjoyment of the exercise program was also high $(6.8 \pm 0.4$, maximum score of 7$)$. Mean score for perceived pain was $5.3 \pm 1.8$, with a maximum score of 7 , indicating participants did not perceive an increase in pain or discomfort during exercise program.

\section{DISCUSSION}

We found that when implemented as a supervised and progressive training program over a defined period of time, the PAG for adults with SCI improve several aspects of physical fitness. Adherence rates for the 16-week exercise program were high and participants were satisfied with the training program.

The improvements observed in peak aerobic capacity and muscle strength are consistent with previous reports. ${ }^{5,6,8-10}$ The increase in submaximal power output performed during the aerobic endurance test in the PAG group indicates that participants were able to perform more work at the same HR and RPE for the same amount of time. Similar results have been reported in previous training studies and are
Table 1 Participant characteristics

\begin{tabular}{lcc}
\hline & $P A G$ & $C O N$ \\
\hline Sample size $(n)$ & 12 & 11 \\
Age (years) & $40.0 \pm 12.3(25-56)$ & $45.9 \pm 11.5(23-65)$ \\
Sex (male/female) & $12 / 0$ & $11 / 2$ \\
Level of injury & C3 $-\mathrm{T} 10$ & $\mathrm{C} 1-\mathrm{T} 11$ \\
Time post injury & $15.0 \pm 8.52(1-36)$ & $9.25 \pm 10.0(1.5-31)$ \\
AIS & A: 2, B: 2, C: $7, \mathrm{D}: 1$ & A: 4, B: $1, \mathrm{C}: 5, \mathrm{D}: 1$
\end{tabular}

Abbreviations: AIS, American Spinal Injury Association Impairment Score; CON, control; PAG, physical activity guidelines.

Values are mean \pm s.d. (range)

likely reflective of increases in both muscle strength and cardiovascular capacity. ${ }^{6,9}$ The most likely mechanisms for the increase in muscle strength include increases in muscle cross-sectional area and neural activation. ${ }^{13}$ While the small pool of related literature makes it difficult to extrapolate the current results to any associated improvements in morbidity risk, the well established links between physical fitness and health in the able-bodied population would suggest that an increase in fitness of this magnitude would be expected to translate to a substantial clinical benefit. ${ }^{14}$

The current recommended minimum amount of aerobic physical activity for able-bodied adults is 150 min per week. ${ }^{15}$ Given that the PAG group in the current study exercised for less than a third of this recommendation (40 min per week), it is clear that improvements in aerobic capacity are possible with a lower threshold of activity in this untrained population. The guidelines for resistance exercise are similar for able-bodied and SCI populations, ${ }^{15}$ and likely appropriate given the limited number of upper body muscles that can be trained following paralysis and the risk of over-use injury.

It has been suggested in other clinical populations that the most critical aspect of an exercise prescription is the training intensity. ${ }^{16}$ The intensity of exercise in the able-bodied population is typically prescribed using percentages of maximum HR. Owing to alterations in the sympathetic control of the heart and a blunted heart rate response to exercise, these methods cannot be relied on following SCI. In this study, we utilized a 10-point RPE scale to prescribe and monitor exercise intensity, instructing participants to train at a value between three and six. On the basis of the improvements in fitness observed, it is evident that, when given the correct instruction and equipment, individuals are able to use RPE to select an appropriate training intensity to improve physical fitness. It is also important to highlight that the current study employed a progressive training program, with increasing time of aerobic exercise and training weight. Although the guidelines serve as an important tool for the general SCI population, a progressive implementation of these guidelines is likely necessary to achieve fitness benefits.

The adherence rate of the CON group was quite variable compared with the PAG group, suggesting that the one-on-one attention during training might have been a significant motivator for participants in the PAG group. There is evidence in support of this; Ditor et al. ${ }^{17}$ reported a significant drop in adherence rates from $80.6 \%$ to $42.7 \% 3$-month following a 9-month training study, where participants were invited to continue exercising at the same facility but were not monitored in the same way as during the intervention period. The high adherence rate in the PAG group can also be attributed to the fact that participation eliminated some of the most commonly reported barriers to exercise participation in the SCI population: knowledge about exercise, facility and equipment accessibility, and personal support. ${ }^{18-20}$ This emphasizes the importance of not only allocating resources into the 
Table 2 Peak aerobic capacity pre and post 4-months of exercise training

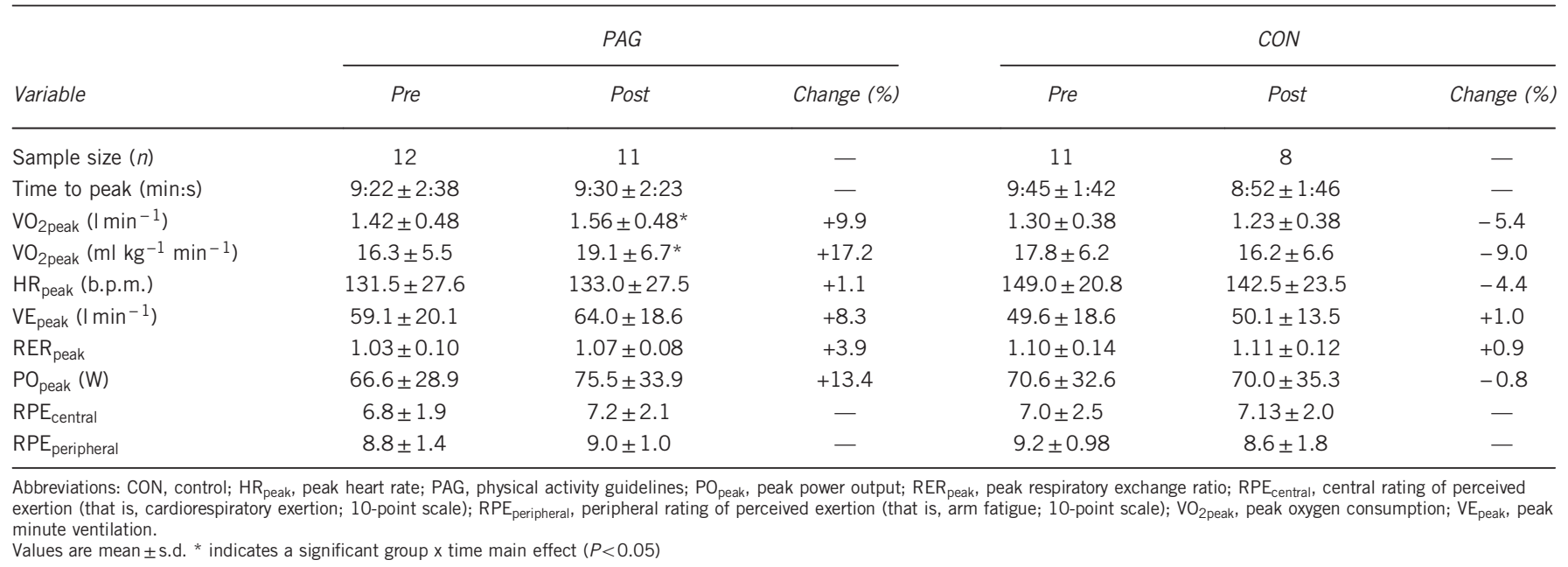

Table 3 Results of aerobic endurance test pre and post 16-weeks of training

\begin{tabular}{|c|c|c|c|c|}
\hline & \multicolumn{2}{|c|}{$P A G$} & \multicolumn{2}{|c|}{ CON } \\
\hline & Pre & Post & Pre & Post \\
\hline Sample size $(n)$ & 11 & 10 & 9 & 8 \\
\hline Power output (W) & $50.9 \pm 21.2$ & $64.3 \pm 21.7^{*}$ & $45.6 \pm 22.3$ & $51.7 \pm 25.0$ \\
\hline Performance time (min:s) & $14: 18 \pm 6: 09$ & $16: 09 \pm 4: 53$ & $16: 06 \pm 5: 33$ & $17: 05 \pm 4: 34$ \\
\hline Steady state HR (b.p.m.) & $121.9 \pm 22.9$ & $131.3 \pm 29.7$ & $131.8 \pm 20.3$ & $131.0 \pm 22.0$ \\
\hline Steady state $\mathrm{VO}_{2}\left(\mathrm{ml} \mathrm{kg}^{-1} \mathrm{~min}^{-1}\right)$ & $15.1 \pm 4.9$ & $17.7 \pm 6.2$ & $13.8 \pm 7.2$ & $15.9 \pm 6.5$ \\
\hline $\mathrm{RPE}_{\text {central }}$ & $6.0 \pm 1.7$ & $6.6 \pm 2.1$ & $6.8 \pm 2.2$ & $6.4 \pm 2.2$ \\
\hline$R P E_{\text {peripheral }}$ & $7.3 \pm 1.4$ & $7.8 \pm 1.4$ & $8.0 \pm 2.5$ & $7.63 \pm 2.5$ \\
\hline
\end{tabular}

Abbreviations: CON, control; $\mathrm{HR}$, heart rate; $\mathrm{HR}_{\text {peak, }}$ peak heart rate; $\mathrm{PAG}$, physical activity guidelines; $\mathrm{RPE}_{\text {central }}$, central rating of perceived exertion (that is, cardiorespiratory exertion; 10 -point scale); RPE $E_{\text {peripheral, }}$ peripheral rating of perceived exertion (that is, arm fatigue; 10-point scale).

$\mathrm{VO}_{2}$, oxygen consumption.

Values are mean \pm s.d. * indicates a significant $(P<0.05)$ group $x$ time interaction

Table 4 Changes in muscle strength (1RM) pre and post 16-weeks of training

\begin{tabular}{|c|c|c|c|c|c|c|}
\hline & \multicolumn{3}{|c|}{$P A G$} & \multicolumn{3}{|c|}{ CON } \\
\hline & Pre & Post & Change (\%) & Pre & Post & Change (\%) \\
\hline Sample size $(n)$ & 12 & 12 & - & 9 & 8 & - \\
\hline Latissimus pull-down & $52.1 \pm 11.6$ & $58.1 \pm 12.8$ & +11.5 & $44.0 \pm 17.3$ & $42.3 \pm 18.0$ & -3.9 \\
\hline Vertical bench press & $45.7 \pm 10.4$ & $52.9 \pm 13.2^{*}$ & +15.8 & $39.3 \pm 24.4$ & $38.2 \pm 25.6$ & -2.8 \\
\hline Seated row & $43.5 \pm 19.5$ & $51.8 \pm 17.6^{*}$ & +19.1 & $41.0 \pm 20.2$ & $40.1 \pm 15.4$ & -2.2 \\
\hline Bicep curl-right & $34.7 \pm 12.7$ & $40.3 \pm 12.8$ & +16.1 & $26.6 \pm 10.7$ & $27.6 \pm 11.3$ & +3.8 \\
\hline Bicep curl-left & $35.6 \pm 10.9$ & $41.6 \pm 13.4$ & +16.9 & $29.2 \pm 8.7$ & $30.3 \pm 9.3$ & +3.8 \\
\hline Rickshaw press & $57.6 \pm 7.4$ & $80.0 \pm 25.0^{*}$ & +38.9 & $61.7 \pm 27.5$ & $62.0 \pm 26.5$ & +0.5 \\
\hline
\end{tabular}

Abbreviations: CON, control; PAG, physical activity guidelines.

Values are mean $(\mathrm{kg}) \pm$ s.d. ${ }^{*}$ indicates a significant $(P<0.05)$ group $\mathrm{x}$ time interaction.

development of community-based exercise programs for people with SCI, but also stresses the value of personal support in order to encourage sustained and effective participation.

\section{Study limitations}

The utilization of an active CON group was both a strength and limitation of this study. Although it is true that an inactive CON group would have provided the best opportunity to evaluate the effectiveness of the PAG, using a control group that was recreationally active allowed us to examine the specific prescription recommendations (related to exercise intensity) associated with the guidelines.

\section{CONCLUSIONS}

The results of this study demonstrate the benefits of implementing the PAG for adults with SCI and show that the guidelines are effective for improving physical fitness. High adherence rates and satisfaction with 
the training program indicate that these guidelines are well received by the consumer and should thus be incorporated into knowledge translation activities. Future studies should now be undertaken to determine if following the guidelines would result in improved cardiovascular health or reduce the risk of secondary morbidities.

\section{DATA ARCHIVING}

There were no data to deposit.

\section{CONFLICT OF INTEREST}

The authors declare no conflict of interest.

\section{ACKNOWLEDGEMENTS}

All authors were funded by mentor trainee awards from the Ontario Neurotrauma Foundation (2009-RHI-MTNI-801; 2011-ONF-RHI-MT-888). Maureen MacDonald has operational support from NSERC (RGPIN 238819-13).

1 Garshick E, Kelley A, Cohen SA, Garrison A, Tun CG, Gagnon D et al. A prospective assessment of mortality in chronic spinal cord injury. Spinal Cord 2005; 43: 408-416.

2 Sweet SN, Martin Ginis KA, Tomasone JR. Investigating intermediary variables in the physical activity and quality of life relationship in persons with spinal cord injury. Health Psychol 2013; 32: 877-885.

3 Ginis KAM, Hicks AL, Latimer AE, Warburton DER, Bourne C, Ditor DS et al. The development of evidence-informed physical activity guidelines for adults with spinal cord injury. Spinal Cord 2011; 49: 1088-1096.

4 Hicks AL, Ginis KAM, Pelletier CA, Ditor DS, Foulon B, Wolfe DL. The effects of exercise training on physical capacity, strength, body composition and functional performance among adults with spinal cord injury: a systematic review. Spinal Cord 2011; 49: 1103-1127.

5 Bizzarini E, Saccavini M, Lipanje F, Magrin P, Malisan C, Zampa A. Exercise prescription in subjects with spinal cord injuries. Arch Phys Med Rehabil 2005; 86: 1170-1175.
6 Bougenot M-P, Tordi N, Betik AC, Martin X, Le Foll D, Parratte B et al. Effects of a wheelchair ergometer training programme on spinal cord-injured persons. Spinal Cord 2003; 41: 451-456.

7 Sabatier MJ, Stoner L, Mahoney ET, Black C, Elder C, Dudley GA et al. Electrically stimulated resistance training in $\mathrm{SCl}$ individuals increases muscle fatigue resistance but not femoral artery size or blood flow. Spinal Cord 2005; 44: 227-233.

8 Jayaraman A, Thompson CK, Rymer WZ, Hornby TG. Short-term maximal-intensity resistance training increases volitional function and strength in chronic incomplete spinal cord injury: a pilot study. J Neurol Phys Ther 2013; 37: 112-117.

9 Hicks AL, Martin KA, Ditor DS, Latimer AE, Craven C, Bugaresti J et al. Long-term exercise training in persons with spinal cord injury: effects on strength, arm ergometry performance and psychological well-being. Spinal Cord 2003; 41: 34-43.

10 Nash MS, van de Ven I, van Elk N, Johnson BM. Effects of circuit resistance training on fitness attributes and upper-extremity pain in middle-aged men with paraplegia. Arch Phys Med Rehabil 2007; 88: 70-75.

11 Pelletier CA, Jones G, Latimer-Cheung AE, Warburton DE, Hicks AL. Aerobic capacity, orthostatic tolerance, and exercise perceptions at discharge from inpatient spinal cord injury rehabilitation. Arch Phys Med Rehabil 2013; 94: 2013-2019.

12 Borg G. Psychophysical bases of perceived exertion. Med Sci Sports Exerc 1982; 14: 377-381.

13 Folland JP, Williams AG. The adaptations to strength training: morphological and neurological contributions to increased strength. Sports Med 2007; 37: 145-168.

14 Myers J, Kaykha A, George S, Abella J, Zaheer N, Lear S et al. Fitness versus physical activity patterns in predicting mortality in men. Am J Med 2004; 117: 912-918.

15 Tremblay MS, Warburton DER, Janssen I, Paterson DH, Latimer AE, Rhodes RE et al. New Canadian physical activity guidelines. Appl Physiol Nutr Metab 2011; 36: 36-46.

16 Moholdt T, Madssen E, Rognmo O, Aamot IL. The highter the better? Interval training intensity in coronary heart disease. J Sci Med Sport 2013; 17: 506-510.

17 Ditor DS, Latimer AE, Martin Ginis KA, Arbour KP, McCartney N, Hicks AL. Maintenance of exercise participation in individuals with spinal cord injury: effects on quality of life, stress and pain. Spinal Cord 2003; 41: 446-450.

18 Rimmer JH, Riley B, Wang E, Rauworth A, Jurkowski J. Physical activity participation among persons with disabilities: barriers and facilitators. Am J Prev Med 2004; 26 : 419-425.

19 Cowan RE, Nash MS, Anderson KD. Exercise participation barrier prevalence and association with exercise participation status in individuals with spinal cord injury. Spinal Cord 2013; 51: 27-32.

20 Scelza WM, Kalpakjian CZ, Zemper ED, Tate DG. Perceived barriers to exercise in people with spinal cord injury. Am J Phys Med Rehabil 2005; 84: 576-583. 